Spin Physics (SPIN2014)

International Journal of Modern Physics: Conference Series

Vol. 40 (2016) 1660100 (10 pages)

(C) The Author(s)

DOI: $10.1142 /$ S2010194516601009

\title{
Review of Polarized Ion Sources
}

\author{
A. Zelenski \\ BNL, Upton,11973, New York, USA \\ zelenski@bnl.gov
}

Published 29 February 2016

\begin{abstract}
Recent progress in polarized ion sources development is reviewed. New techniques for production of polarized $\mathrm{H}^{-}$ion (proton), $\mathrm{D}^{-}\left(\mathrm{D}^{+}\right)$and ${ }^{3} \mathrm{He}^{++}$ion beams will be discussed. A novel polarization technique was successfully implemented for the upgrade of the RHIC polarized $\mathrm{H}^{-}$ion source to higher intensity and polarization. In this technique, a proton beam inside the high magnetic field solenoid is produced by ionization of the atomic hydrogen beam (from an external source) in the He-gas ionizer cell. Polarized electron capture from the optically-pumped $\mathrm{Rb}$ vapor further produces proton polarization (Optically Pumped Polarized Ion Source technique). The upgraded source reliably delivered beam for the 2013 polarized run in RHIC at $\sqrt{S}=510 \mathrm{GeV}$. This was a major factor contributing to RHIC polarization increase to over $60 \%$ for colliding beams. Feasibility studies of a new polarization technique for polarized ${ }^{3} \mathrm{He}^{++}$source based on BNL Electron Beam Ion Source is also discussed.
\end{abstract}

Keywords: Polarized source; Polarized beam; Polarized ${ }^{3} \mathrm{He}$ source.

PACS numbers: $34.10 ; 34.70 ; 34.80$

\section{Introduction}

Polarization is an intrinsic property of photons, electrons, protons, nuclear beams, and the study of polarization effects provides essential information on particle structure and their interactions. Collider experiments with polarized beams at RHIC and HERA (at HERA, the experimental program with polarized electron beam and polarized internal target, HERMES, has been completed) provide tests of QCD and Electroweak interaction. Polarization asymmetries and parity violation are strong signatures for the identification of the fundamental processes, which are otherwise inaccessible. Such experiments require the maximum available luminosity, and therefore polarization should be obtained as an extra beam quality without sacrificing intensity. High intensity polarized $\mathrm{H}^{-}$ion sources are presently a common choice for high-energy accelerators due to the advantage of stripping injection into

This is an Open Access article published by World Scientific Publishing Company. It is distributed under the terms of the Creative Commons Attribution 3.0 (CC-BY) License. Further distribution of this work is permitted, provided the original work is properly cited. 


\section{A. Zelenski}

the accelerator ring. Polarized deuteron beam will be required for the deuteron EDM (Electron Dipole Moment) experiment and for the Dubna "Nuclotron" facility. Experiments with accelerated polarized ${ }^{3} \mathrm{He}^{++}$ion beams will be a part of the program at future Electron Ion Collider.

\section{Polarization Techniques}

Spin filtering techniques. The basic feature of these polarization techniques is an attenuation: scattering out, adsorption, defocusing, quenching of unwanted part of light, electron, atomic, proton, or nuclei beams having 'unwanted' direction of polarization. A strong spin dependence of thermal energy neutron beam capture in a polarized ${ }^{3} \mathrm{He}$ gas cell is often used for neutron beam polarization and polarization analysis. In Lamb-shift polarized sources the metastable hydrogen atoms in unwanted spin states are quenched by the "spin-filter" to the ground states and remaining polarized atoms can be produced by selective ionization from metastable states.

Selective focusing by sextupole separating magnets is used for hydrogen (deuterium) beam polarization by electron spin in Atomic Beam Sources (ABS) of polarized ions and polarized internal targets. In these sources the atomic hydrogen is produced by dissociation of hydrogen molecules in RF discharge. Hydrogen gas flows out the dissociator volume to vacuum forming gaseous jet. The atomic hydrogen beam is formed then from central part of the jet using skimmers and diaphragms. A typical velocity of atoms in the beam is about $(1-2) \cdot 10^{5} \mathrm{~cm} / \mathrm{s}$, which is achieved by cooling of the dissociator nozzle to a temperature of 30-80 K. The sextupole magnetic field acts on electron magnetic moment axially aligned with the field gradient towards magnet tips as a focusing lens. Then the electron polarization transferred to the protons by means of radio-frequency transitions. The atomic beam of a selected spin-state directed into an ionizer (or a storage cell). The polarized proton or $\mathrm{H}^{-}$ ion beam can be produced by ionization of atomic beam in magnetic field of about $1.5 \mathrm{kG}$, which is sufficient to break electron-proton spin coupling while preserving proton polarization. The ABS beam can be used as an internal target in collider storage ring (polarized $\mathrm{H}^{-}$jet polarimeter at $\mathrm{RHIC}^{1}$ ), or for feeding storage cell type of internal targets. There is a proposal to use polarized atomic hydrogen storage cell for anti-proton polarization in the storage ring by filtering (removing) of unwanted states (PAX proposal for HESR at FAIR ${ }^{2}$ ).

Optical pumping. Electron or nuclear polarized atoms can be obtained in process of absorption of polarized photons from external source of polarized light and subsequent spontaneous emission of un-polarized photon (optical pumping). The electron polarization is transferred to protons by spin-spin interaction if optical pumping takes place in sufficiently low magnetic field. Population of spin states of atoms is changed during the optical pumping without filtering process due to absorption of external polarized photons which angular momentum is transmitted to atoms. 
Polarization-transfer technique. In any type of polarized proton $\left(\mathrm{H}^{-}\right.$ion $)$source the first step is the generation of an electron-spin polarized atomic beam. The polarization then transferred to the protons by hyperfine interaction and finally the beam is ionized. The difference is in the velocity of the atomic beam. It is comparatively easy to polarize a "slow" (thermal energy) beam by using separating magnets, as discussed above, but there is a basic limitation of about (1-2)10 17 atoms/s on atomic beam intensity. The advantages of using "fast" (a few keV energy) beams are higher intensity and simple, more efficient ionization. In this technique optical pumping is used to get polarized alkali atoms. The electron-spin polarization of the "fast" $\mathrm{H}$ beam is produced either in a charge-exchange process, when primary protons capture polarized electrons from polarized atoms in a vapor cell, or in spin-exchange collisions. This technique is called an "Optically-Pumped Polarized Ion Source" (OPPIS), although polarized electrons can also be captured from a ferromagnetic foil (as in the original Zavoiski's proposal), or from hydrogen, or an alkali-metal atomic beam polarized by separating magnets. ${ }^{3}$

\section{Atomic Beam Source with Resonant Plasma Ionizer}

A polarized ion source with resonant plasma ionizer developed at INR Moscow. ${ }^{4}$ A deuterium plasma injector used in this source for production of polarized $\mathrm{H}^{-}$ ions. The injector generates plasma consisting mainly from $\mathrm{D}^{+}$and $\mathrm{D}^{-}$ions. The plasma flux from the arc-discharge plasma source is enriched by negative ions in a surface-plasma converter. Positive ions converted into neutral atoms with eV energy in collisions with a neutralizer internal surface. Polarized atomic hydrogen beam is injected into the plasma and polarized $\mathrm{H}^{-}$ions are produced via reaction: $\mathbf{H}^{0} \uparrow+$ $\mathbf{D}^{-} \Rightarrow \mathbf{H}^{-\uparrow}+\mathbf{D}^{0}$. With this ionizer a polarized $\mathrm{H}^{-}$ion beam with peak current of $4 \mathrm{~mA}$ has been obtained with $\mathrm{D}^{-}$ion beam current of $62 \mathrm{~mA}$. The polarization of the $\mathrm{H}^{-}$ion beam was measured to be $0.91 \pm 0.03$. The Lamb shift polarimeter has been used for the polarization measurements. Efficiency of direct conversion of polarized hydrogen atoms into polarized $\mathrm{H}^{-}$ions reached of $12.5 \%$. Polarized $\mathrm{D}^{-}$ ion beam with peak intensity up to $2 \mathrm{~mA}$ and polarization up to $90 \%$ from nominal of vector polarization \pm 1 and tensor polarization of $+1,-2$ have been obtained from the polarized ion source CIPIOS at IUCF. ${ }^{5}$ The source was developed in a collaboration of IUCF and INR Moscow. The source had a nearly resonant chargeexchange plasma ionizer and produced also polarized $\mathrm{H}^{-}$ions $(1.8 \mathrm{~mA}$ peak) and un-polarized $\mathrm{H}^{-}$and $\mathrm{D}^{-}$ion beams (40 $\mathrm{mA}$ and $30 \mathrm{~mA}$ respectively) with pulse duration of $300 \mu \mathrm{s}$ and repetition rate of $2 \mathrm{~Hz}$.

The program of polarization research at Joint Institute for Nuclear Research at Dubna, Russia based on the acceleration of polarized deuteron beams up to 10 GeV beam energy in the NUCLOTRON accelerator. A high intensity pulsed source of polarized deuterons is required to achieve the number of accelerated deuterons of $\sim 10^{10}$ deuterons/cycle (with the present one turn injection scheme, $10 \mu$ s pulse duration. $1 \mathrm{~Hz}$ repetition rate). A new polarized deuteron source is being built in 


\section{A. Zelenski}

a collaboration of JINR and INR Moscow. A project goal is a polarized deuteron beam from the source with a peak intensity of $10 \mathrm{~mA}$ and polarization of $90 \%$ from nominal vector polarization \pm 1 and tensor polarization $+1,-2$. A nearly resonant charge-exchange deuteron plasma ionizer with storage cell will be used to increase intensity of the polarized ion beam from the source. ${ }^{6}$

\section{Polarized Source with Cesium Beam Ionizer at COSY}

The principle of the source is an ionization of pulsed polarized hydrogen or deuterium beams (20 ms pulse duration, $0.5 \mathrm{~Hz}$ repetition rate) in collisions with a pulsed neutral cesium beam having a kinetic energy of about $45 \mathrm{keV}{ }^{7}$ The Cs ion emitter is a porous tungsten button on a molybdenum heater. The pulsed operation of the Cesium gun controlled via a high voltage electrode. After the $\mathrm{Cs}^{+}$beam formation to match the polarized hydrogen beam in the ionizing region the $\mathrm{Cs}^{+}$ beam is neutralized in the cesium neutralizer cell. In a charge exchange reaction is taking place in a solenoid field, negatively charged hydrogen, or deuteron, ions are created and accelerated toward the extraction elements. Then the ions are bent magnetically by $90^{\circ}$, passed through a Wien-filter and enter the transporting source beam line that guides them into the cyclotron. The new record value of $50 \mu \mathrm{A}$ and $90 \%$ polarization was reached during routine source operation in 2005 . This exceeds the original design value of $30 \mu \mathrm{A}$. It was the result of the optimization of all source components. $^{8}$

\section{Optically Pumped Polarized $\mathbf{H}^{-}$Ion Source at RHIC}

A novel polarization technique was successfully implemented for the upgrade of the RHIC polarized $\mathrm{H}^{-}$ion source to higher intensity and polarization. In this technique, a proton beam inside the high magnetic field solenoid is produced by ionization of the atomic hydrogen beam (from an external source) in the He-gas ionizer cell. Proton polarization is produced by the process of polarized electron capture from the optically-pumped Rb vapor. Polarized beam intensity is produced in the source exceeds $4.0 \mathrm{~mA}$. Strong space-charge effects cause significant beam losses in the LEBT (Low Energy Beam Transport, $35.0 \mathrm{keV}$ beam energy) line. The LEBT was modified to reduce losses. As a result, $1.4 \mathrm{~mA}$ of polarized beam was transported to the RFQ and $0.7 \mathrm{~mA}$ was accelerated in linac to $200 \mathrm{MeV}$. The upgraded source reliably delivered beam for the 2013 polarized run in RHIC at $\sqrt{S}=510 \mathrm{GeV}$. This was a major factor contributed to the RHIC polarization increase to over $60 \%$ for colliding beams.

OPPIS upgrade with the Atomic Hydrogen Beam Injector. The polarized beam for the RHIC spin physics experimental program is produced in the OpticallyPumped Polarized $\mathrm{H}^{-}$Ion Source (OPPIS). ${ }^{9}$ An Electron Cyclotron Resonance (ECR) ion source was used as the primary proton source in the old operational 
polarized source. The ECR source was operated in a high magnetic field. The proton beam produced in the ECR source had a comparatively low emission current density and high beam divergence. In pulsed operation, suitable for application at high-energy accelerators and colliders, the ECR source limitations can be overcome by using a high brightness proton source outside the magnetic field instead of the ECR source. In this technique (which was implemented for the first time at INR, Moscow ${ }^{10}$ ), the proton beam is focussed and neutralized in a hydrogen cell producing the high brightness $6.0-8.0 \mathrm{keV}$ atomic $\mathrm{H}^{0}$ beam. The atomic $\mathrm{H}^{0}$ beam is injected into the superconducting solenoid, where both the He ionizer cell and the opticallypumped $\mathrm{Rb}$ cell are situated in the $25-30 \mathrm{kG}$ solenoid field. The solenoid field is produced by a new superconducting solenoid with a re-condensing cooling system. The injected $\mathrm{H}$ atoms are ionized in the He cell with $60-80 \%$ efficiency to form a low emittance intense proton beam and then enter the polarized $\mathrm{Rb}$ vapour cell (see Fig. 1). The protons pick up polarized electrons from the Rb atoms to become a beam of electron-spin polarized $\mathrm{H}$ atoms (similar to the ECR based OPPIS). A negative bias of about $3.0-5.0 \mathrm{kV}$ applied to the He cell decelerate the proton beam produced in the cell to the 2.0-3.0 keV beam energy, optimal for the charge-exchange collisions in the rubidium and sodium cells. This allows energy separation of the polarized hydrogen atoms produced after lower energy proton neutralization in Rbvapour and residual hydrogen atoms of the primary beam.

Fast atomic beam source development. In the atomic hydrogen beam source the primary proton beam is produced by a four-grid multi-aperture ion extraction optical system and neutralized in the $\mathrm{H}_{2}$ gas cell downstream from the grids. A highbrightness atomic hydrogen beam was obtained in this injector by using a plasma emitter with a low transverse ion temperature (of about $0.2 \mathrm{eV}$ ), which is formed by plasma jet expansion from the arc plasma generator. The multi-hole grids are

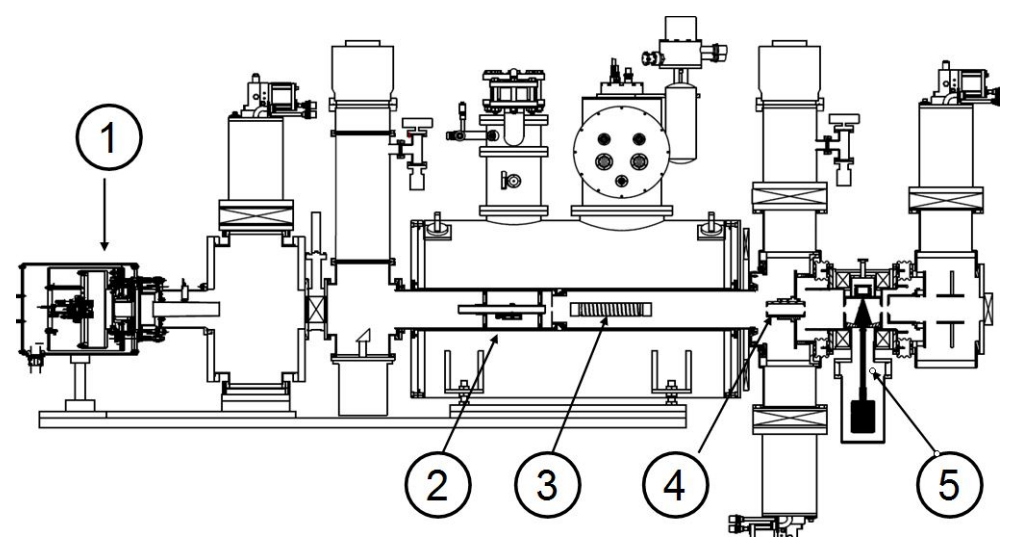

Fig. 1. A new polarized source layout: 1-atomic hydrogen injector; 2- pulsed He-gaseous ionizer cell; 3-optically-pumped Rb-vapour cell; 4-Sona-transition; 5-Na-jet ionizer cell. 


\section{A. Zelenski}

spherically shaped to produce "geometrical" beam focusing. The grids are made of $0.4 \mathrm{~mm}$ thick molybdenum plates. Holes $(0.8 \mathrm{~mm}$ diameter $)$ in the plates were produced by photo-etching techniques. The hole array form a hexagonal structure with a step of $1.1 \mathrm{~mm}$ and outer diameter of $5.0 \mathrm{~cm}$. The grids were shaped by re-crystallization under pressure at high temperature and were welded to stainless steel holders by a pulsed $\mathrm{CO}_{2}$ laser. At an emission current density of $470 \mathrm{~mA} / \mathrm{cm}^{2}$, the angular divergence of the produced beam was measured to be $\sim 10-12 \mathrm{mrad}$.

The focal length of the spherical ion extraction system was optimized for the OPPIS application, which is characterized by a long polarizing structure of the charge-exchange cells and small $(2.0 \mathrm{~cm}$ diameter) Na-jet ionizer cell, which is located $240 \mathrm{~cm}$ from the source (see Fig. 1). An optimal drift-space length of about $140 \mathrm{~cm}$ is required for convergence of the $5 \mathrm{~cm}$ (initial diameter) beam to $2.5 \mathrm{~cm}$ diameter He-ionizer cell. About $20 \%$ of the total beam intensity ( $23.5 \mathrm{~A})$ can be transported through the Na-jet cell acceptance by using the optimal extraction grid system with a focal length: $F \sim 200 \mathrm{~cm}$. Three spherical IOS were tested on the test-bench at BNL. The focusing lengths of IOS \#1 and \#3 were $\sim 150 \mathrm{~cm}$ and for IOS\#3 F $250 \mathrm{~cm}$, which allowed study of optimal beam formation. IOS\#2 produced about $500 \mathrm{~mA}$ equivalent atomic $\mathrm{H}$ beam within the $2.0 \mathrm{~cm}$ diameter Na-jet ionizer acceptance (at the distance $240 \mathrm{~cm}$ from the source) and $16 \mathrm{~mA} \mathrm{H}^{-}$ion beam current. ${ }^{11}$

Helium ionizer cell. Beam energy separation. The He-ionizer cell is a $40 \mathrm{~cm}$ long stainless steel tube with an inside diameter $25.4 \mathrm{~mm}$. A new fast "electromagnetic" valve for He-gas injection to the cell was developed for operation in the $30 \mathrm{kG}$ solenoid field. In this valve, a pulsed current of about $100 \mathrm{~A}$ is passed through the flexible springing plate (made of beryllium bronze foil with a thickness of 0.5 $\mathrm{mm})$. The Lorentz force: $\mathbf{F}=\mathrm{eL}[\mathbf{I} \times \mathbf{B}]=15 \mathrm{~N}$ for a $\mathrm{L}=5 \mathrm{~cm}$ long plate. The plate is fixed at one end and this force bends the plate and opens the small $(0.5 \mathrm{~mm}$ diameter) hole which is sealed with a Viton O-ring. The pulsed current rise-time is $\sim 50 \mu$ s and gas pressure rise time is about $100 \mu \mathrm{s}$.

The proton beam produced in the He cell is decelerated from $6.5 \mathrm{keV}$ to $2.5 \mathrm{keV}$ by a negative potential of $4.0 \mathrm{keV}$ applied to the cell. At the $2.5 \mathrm{keV}$ beam energy, the $\mathrm{H}^{-}$ion yield in the sodium ionizer cell is near maximum $(\sim 8.4 \%)$ and the polarized electron capture cross-section from $\mathrm{Rb}$ atoms is also near the maximum of $\sim 0.8 \cdot 10^{-14} \mathrm{~cm}^{2}$. The deceleration was produced by a precisely aligned (to reduce beam losses) three wire-grid system. A negative bias applied to the first grid at the cell entrance and second grid at the cell exit to trap electrons in the cell for space-charge compensation.

About $40 \%$ residual (which passed the He-cell without ionization) atomic beam component at $6.5 \mathrm{keV}$ energy will pass through the deceleration system and Rb cell and be ionized in Na-cell producing $\mathrm{H}^{-}$ion beam. The $\mathrm{H}^{-}$ion beam acceleration produce polarized $\mathrm{H}^{-}$ion beam with $35 \mathrm{keV}$ beam energy and un-polarized beam with $39.5 \mathrm{keV}$ energy. The un-polarized $39.5 \mathrm{keV}$ beam component is well separated 
after the 23.7 degree bending magnet in the LEBT. In measurements of beam separation, the beam energy was varied by the accelerating voltage applied to the Na-jet ionizer cell. The residual $6.5 \mathrm{keV}$ un-polarized beam component is strongly suppressed (to less than $2 \%$ of polarized beam component).

\section{Experimental Results}

The beam polarization was measured in the precision absolute polarimeter at 200 $\mathrm{MeV}$ beam energy after the Linac. ${ }^{12}$ The polarization and $\mathrm{H}^{-}$beam current measurements (after acceleration to $200 \mathrm{MeV}$ in the Linac) vs. Rb-vapor thickness presented in Fig. 2.

At low Rb-vapor densities, the residual (un-polarized) $\mathrm{H}^{-}$ion beam current produced by neutralization in residual gas and incomplete energy separation is less than $0.01 \mathrm{~mA}$. Therefore, the polarization dilution due to these factors does not exceed $\sim 2 \%$. There was observed some polarization drop at $\mathbf{N L} \geq 10^{14}$ atoms $/ \mathrm{cm}^{2}$ $\left(\mathbf{N} \sim 3 \cdot 10^{13}\right.$ atoms $/ \mathrm{cm}^{3}$, Rb-cell length $\left.-\mathbf{L}=30 \mathrm{~cm}\right)$. Depolarization due to "radiation trapping" is small (at this density Rb-vapor), but some polarization losses may occur due to reduced polarization near the cell walls. This limits the proton beam size and requires good matching between the beam and the Rb-cell diameter. These losses depend on atomic beam intensity and reduced by laser tuning and atomic beam parameters optimization.

The $\mathrm{H}^{-}$ion beam produced in the sodium-jet ionizer cell is accelerated to 35 $\mathrm{keV}$. This energy increase is essential for the high intensity beam transport. Strong space-charge effects cause significant beam losses in the LEBT line. Basic limitations on the high-intensity polarized $\mathrm{H}^{-}$ion beam production and transport were

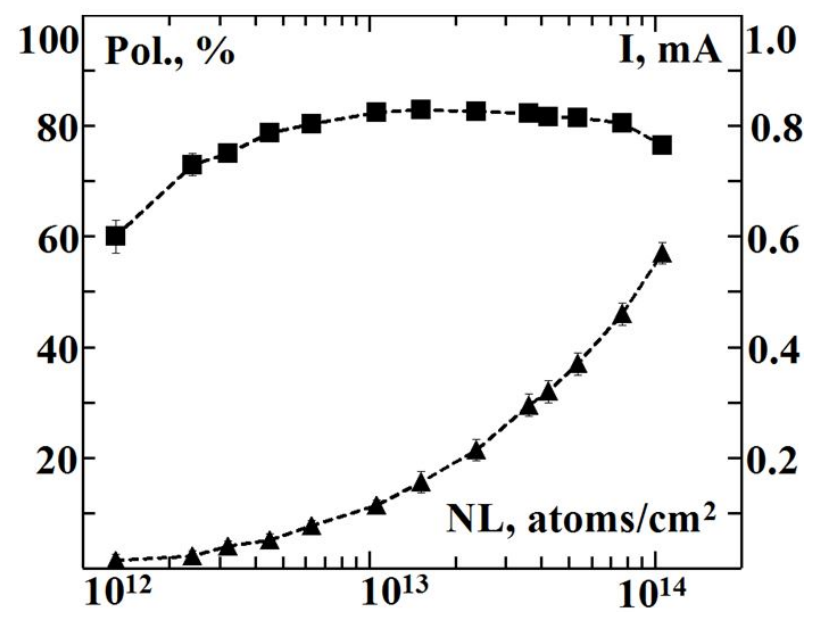

Fig. 2. Polarization (squares at the left axis) and beam intensity (triangles- in $\mathrm{mA}$ at the right axis) vs. Rb-vapor thickness. 


\section{A. Zelenski}

experimentally studied in charge-exchange collisions of the neutral atomic hydrogen beam in the Na-vapor-jet ionizer cell. The LEBT was modified to reduce losses and $1.4 \mathrm{~mA}$ of polarized beam was transported to the RFQ and $\sim 0.7 \mathrm{~mA}$ of beam was accelerated in the Linac to $200 \mathrm{MeV}$. This current can be further increased by increases in the atomic beam intensity and reduction of the beam losses in LEBT.

Electron depolarization due to spin-orbital interaction in exited $(2 \mathrm{~S}, 2 \mathrm{P})$ states of hydrogen atoms should be suppressed by high magnetic field in the Rb-cell. Polarization dependence on magnetic field in the Rb-cell is presented in Fig. 3. At every field, the Sona-transition field distribution was optimized by correction coil tuning. The polarization saturation was observed at super-conducting solenoid fields $\geq 25 \mathrm{kG}$.

In the first year of operation, the new source performance exceeded the old ECRbased source parameters. This was a major factor contributing to the polarization increase to about $60 \%$ for the RHIC colliding beams.

\section{Polarized ${ }^{3} \mathrm{He}^{++}$Source Development for eRHIC}

Polarized beams of ${ }^{3} \mathrm{He}^{++}$ions also contain the polarized neutron component and its magnetic moment is close to the proton magnetic moment value, therefore the AGS and RHIC Siberian snake should preserve polarization during acceleration. ${ }^{13}$ In this case the difficulty is the polarized ${ }^{3} \mathrm{He}^{++}$source. The proposed polarized ${ }^{3} \mathrm{He}^{++}$acceleration in RHIC (and also for future RHIC upgrade to electron ion collider eRHIC) will require about $2 \cdot 10^{11}$ ions in the source pulse and about $10^{11}$ ions in the RHIC bunch. To deliver this intensity in a $20 \mu$ s pulse duration for injection to Booster, the source peak current has to be about $1000 \mu \mathrm{A}$, which is 1000 higher than ever achieved in existed ${ }^{3} \mathrm{He}^{++}$ion sources. A new technique has been proposed for production of high intensity ${ }^{3} \mathrm{He}^{++}$ion beam. It is based on ionization

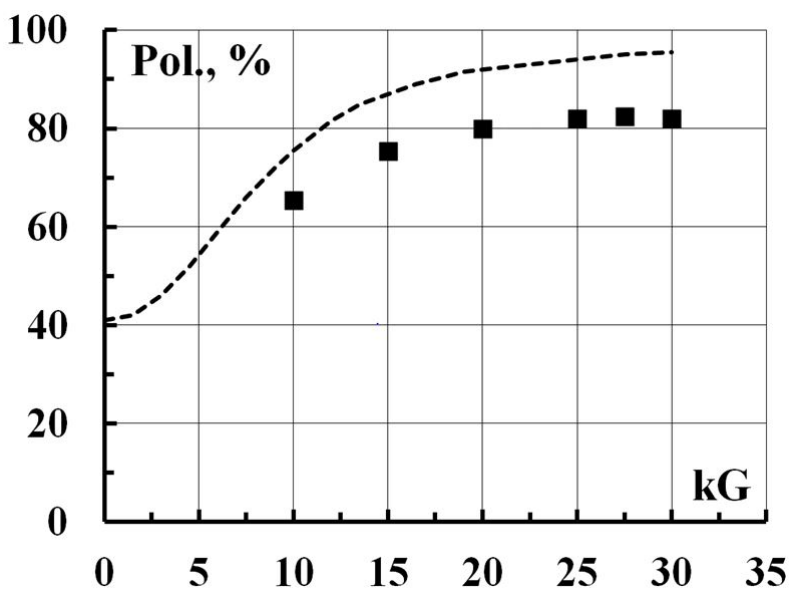

Fig. 3. Polarization vs. magnetic field in the Rb-cell (squares). Dashed line-calculations. 


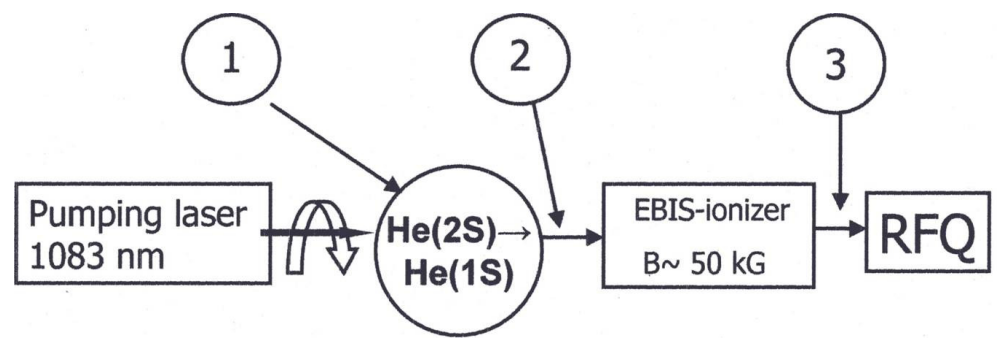

Fig. 4. Schematic diagram of the polarized ${ }^{3} \mathrm{He}^{++}$ion source: 1 - metastability-exchange polarizing cell; 2-polarized ${ }^{3} \mathrm{He}$ transfer line; $\sim 3 \cdot 10^{12}$ polarized ${ }^{3} \mathrm{He}^{++}$ions for injection to RFQ.

of ${ }^{3} \mathrm{He}$ gas (polarized by metastability exchange technique) in the Electron Beam Ion Source (EBIS) ${ }^{14}$ (see Fig. 4). The development of the source for eRHIC is now in progress in collaboration between BNL and MIT. ${ }^{15}$

The highest ${ }^{3} \mathrm{He}$ nuclear polarization in excess of $80 \%$ was achieved by the metastability exchange technique. In this method, ${ }^{3} \mathrm{He}$ gas at typically 1 torr pressure is contained in a glass bulb and a weak RF discharge is maintained in the gas. Metastable atoms in the $2^{3} \mathrm{~S}_{1}$ state are produced in the discharge and may be polarized by means of optical pumping with circularly polarized $\left(2^{3} \mathrm{~S}_{1}-2^{3} \mathrm{P}_{0}\right)$ $1083 \mathrm{~nm}$ light. In the proposed technique, the polarized ${ }^{3} \mathrm{He}$ atoms consumption for injection to an ionizer is very small, of the order of $10^{15} \mathrm{He}$ atoms/s and high polarization is expected. The initial tests at BNL shoved significant depolarization due to magnetic field gradients in the injection transfer line.

There is a possibility of ${ }^{3} \mathrm{He}$ polarization in the high magnetic field. High magnetic field suppresses depolarization and higher polarization was obtained at ${ }^{3} \mathrm{He}$ gas pressure of a 6 torr in the solenoid of a 4.7 T field. ${ }^{16}$ The ${ }^{3}$ He gaseous cell can be placed inside the EBIS solenoid and pulsed gas valve similar to one used in the OPPIS can be used for the gas injection to center of the EBIS drift tube system to minimize depolarization and increase ionization efficiency. The ionization in the EBIS is produced in a $5.0 \mathrm{~T}$ magnetic field, which preserves the nuclear ${ }^{3} \mathrm{He}$ polarization while in the intermediate single-charged ${ }^{3} \mathrm{He}^{+}$state. The number of ions is limited to the maximum charge, which can be confined in the EBIS. From experiments with $\mathrm{Au}^{32+}$ ion production, one expects about $2.5 \cdot 10^{11}{ }^{3} \mathrm{He}^{++}$ions/pulse to be produced and extracted for subsequent acceleration and injection to RHIC. After ${ }^{3} \mathrm{He}^{++}$acceleration to a few $\mathrm{MeV} /$ nucleon, ${ }^{3} \mathrm{He}-\mathrm{D}$ or ${ }^{3} \mathrm{He}-\mathrm{Carbon}$ collisions can be used for polarization measurements.

A study of limitation on the maximum nuclear polarization in the metastability exchange technique (at the very low polarized ${ }^{3} \mathrm{He}$ gas consumption rate) will be required to define the maximum attainable polarization. The study of possible depolarization effects during polarized ${ }^{3} \mathrm{He}$ gas injection to existing EBIS prototype and multi-step ionization process is in progress. The expected ${ }^{3} \mathrm{He}^{++}$ion beam intensity is about $2 \cdot 10^{11}$ ions/pulse with polarization of a $70 \%$. 


\section{Summary}

There are a number of proposals on polarization studies with polarized ion beams at new and existing accelerators and colliders, which will require high-intensity, high polarization proton, deuteron and ${ }^{3} \mathrm{He}^{++}$ion beams. State-of-the-art atomic beam sources with resonant plasma ionizer and optically pumped polarized proton sources produce sufficient beam intensity (of a few $\mathrm{mA} \mathrm{H}^{-}$ion beam intensity) for charging the high-energy accelerators to full capacity (for colliders the intensity is limited by the beam-beam interaction). The proton polarization of about $85-90 \%$ achieved for the high intensity beams. For the polarized ${ }^{3} \mathrm{He}^{++}$source, feasibility studies of the proposals based on EBIS ionizer and resonant charge-exchange are in progress.

\section{References}

1. A. Zelenski, W. Haeberli, Y. Makdisi, A. Nass, J. Ritter, T. Wise, V. Zubets, Nucl. Instrum. Methods A536, 248 (2005).

2. Antiproton-Proton Scattering Experiments with Polarization, PAX Proposal for HESR at FAIR, Julich 2005, e-print Archive: hep-ex/0505054 (2005).

3. A. Zelenski, in Proc. SPIN2000, AIP Conf. Proc., 570, p. 179 (2000).

4. A. Belov, in AIP Conf. Proc., 980, p. 209 (2008).

5. V. P. Derenchuk, A. S. Belov, in AIP Conf. Proc., 675, p. 887 (2003).

6. V. V. Fimushkin, A. S. Belov, A. D. Kovalenko, L. V. Kutuzova, Yu. V. Prokofichev, in Status of the polarized ion source for the JINR accelerator complex, these Conference.

7. W. Haeberli, Nucl. Instrum. Methods 62, p. 355 (1968).

8. R.Gebel, O.Polden, R. Maier, in PSTP 2007, AIP Conf. Proc., 980, p. 231 (2008).

9. A. Zelenski, J. Alessi, G. Dutto, S. Kokhanovski, V. Klenov, Y. Mori, P. Levy et al., Rev. Sci. Instrum. 73, p. 888 (2002).

10. A. Zelenski, S. Kokhanovski, V. Lobashev, V. Polushkin, Nucl. Instrum. Methods A245, p. 223 (1986).

11. A. Kolmogorov, G. Atoian, V. Davydenko, A. Ivanov, J. Ritter, N. Stupishin and A. Zelenski, Rev. Sci. Instrum. 85(2), 02A734 (2014).

12. A. Zelenski et al., in SPIN 2010 Conf.Proc., J. Phys. Conf. Ser., 295012132 (2011).

13. W. MacKay, "Prospects for Acceleration of D and He beams", in AIP Conf. Proc., 980, p. 191 (2008).

14. A. Zelenski and J. Alessi, "Prospects on high intensity optically-pumped polarized $\mathrm{H}^{-}, \mathrm{D}^{-}$and ${ }^{3} \mathrm{He}^{++}$ions", ICFA Beam Dynamics Newsletter No. 30, p. 39 (2003).

15. J. Maxwell et al., see talk in this Conference.

16. C. Talbot, M. Batz, P. Nacher and G. Tastevin, in J. Physics: Conf. Series, 294, 012008 (2011). 